\title{
Object substitution masking in schizophrenia: an event-related potential analysis
}

\author{
1 Veterans Affairs Greater Los Angeles Healthcare System, Los Angeles, CA, USA \\ ${ }^{2}$ Semel Institute for Neuroscience and Human Behavior, University of California, Los Angeles, Los Angeles, USA \\ ${ }^{3}$ San Francisco Veterans Affairs Medical Center, San Francisco, CA, USA \\ ${ }^{4}$ Department of Psychiatry, University of California San Francisco, San Francisco, CA, USA \\ ${ }^{5}$ Department of Psychology, University of Houston, Houston, TX, USA
}

Jonathan K. Wynn ${ }^{1,2}{ }^{*}$, Kristopher I. Mathis ${ }^{1}$, Judith Ford ${ }^{3,4}$, Bruno G. Breitmeyer ${ }^{5}$ and Michael F. Green ${ }^{1,2}$

\section{Edited by:}

Steven Silverstein, University of Medicine and Dentistry of New Jersey, USA

\section{Reviewed by:}

Steven Silverstein, University of Medicine and Dentistry of New Jersey, USA

Anne Giersch, Institut National de la Santé et de la Recherche Médicale, France

\section{*Correspondence:}

Jonathan K. Wynn, Veterans Affairs Greater Los Angeles Healthcare System, Mental IIIness Research, Education, and Clinical Center, Building 210, 11301 Wilshire Boulevard, Los Angeles, CA 90073 , USA.

e-mail: jkwynn@ucla.edu

\begin{abstract}
Schizophrenia patients exhibit deficits on visual processing tasks, including visual backward masking, and these impairments are related to deficits in higher-level processes. In the current study we used electroencephalography techniques to examine successive stages and pathways of visual processing in a specialized masking paradigm, four-dot masking, which involves masking by object substitution. Seventy-six schizophrenia patients and 66 healthy controls had event-related potentials (ERPs) recorded during four-dot masking. Target visibility was manipulated by changing stimulus onset asynchrony (SOA) between the target and mask, such that performance decreased with increasing SOA. Three SOAs were used: 0 , 50 , and $100 \mathrm{~ms}$. The P100 and N100 perceptual ERPs were examined. Additionally, the visual awareness negativity (VAN) to correct vs. incorrect responses, an index of reentrant processing, was examined for SOAs 50 and $100 \mathrm{~ms}$. Results showed that patients performed worse than controls on the behavioral task across all SOAs. The ERP results revealed that patients had significantly smaller P100 and N100 amplitudes, though there was no effect of SOA on either component in either group. In healthy controls, but not patients, N100 amplitude correlated significantly with behavioral performance at SOAs where masking occurred, such that higher accuracy correlated with a larger N100. Healthy controls, but not patients, exhibited a larger VAN to correct vs. incorrect responses. The results indicate that the N100 appears to be related to attentional effort in the task in controls, but not patients. Considering that the VAN is thought to reflect reentrant processing, one interpretation of the findings is that patients' lack of VAN response and poorer performance may be related to dysfunctional reentrant processing.
\end{abstract}

Keywords: visual processing, schizophrenia, ERP, visual backward masking, reentrant processing

\section{INTRODUCTION}

Patients with schizophrenia exhibit several visual processing impairments (Green et al., 1994; Butler et al., 2003; Butler and Javitt, 2005; Rassovsky et al., 2005; Wynn et al., 2005; Silverstein and Keane, 2011), and these impairments have been tied to specific neural abnormalities, such as magnocellular pathway dysfunction, NMDA functioning, and activation in the lateral occipital complex (Butler et al., 2005; Green et al., 2009; Javitt, 2009). However, it is unclear whether visual processing impairment is isolated to early or later processing stages (Javitt, 2009; Dias et al., 2011; Rassovsky et al., 2011). Visual processing divides roughly into two stages: (1) initial formation of a percept, and (2) subsequent processing of the percept until it reaches awareness (Di Lollo et al., 2000; Enns and Di Lollo, 2000; Ro et al., 2003; Enns, 2004; Chen and Treisman, 2009; Dux et al., 2010). The first stage is thought to involve a neural feedforward sweep from retina to primary visual cortex. The second stage involves reentrant processes (i.e., recurrent cortico-cortical circuits) that refine the initially ambiguous percept until it becomes recognizable. These reentrant processes have even been shown to feedback to very early neural areas such as the lateral geniculate nucleus (Sillito et al., 1994).

Visual backward masking can be used to assess both of these stages. Masking that disrupts feedforward processing usually occurs between 0 and $100 \mathrm{~ms}$ after target onset. Masking at the reentrant stage occurs slightly later (starting approximately $100 \mathrm{~ms}$ after stimulus onset; Woodman and Luck, 2003; Prime et al., 2011), and results in the mask displacing the neural representation of the target, a phenomenon termed object substitution masking (Di Lollo et al., 2000; Enns and Di Lollo, 2000; Chen and Treisman, 2009; Dux et al., 2010). The premise underlying object substitution is that the feedforward sweep of the target must be refined at higher cortical levels through reentrant processes to the lower input levels. If the mask is presented before the target is identified at this higher-level, there will be a mismatch between the feedforward and reentrant sweeps, and processing will switch to the mask. In essence, the target may be correctly identified at some level within the primary visual processing stream but accurate information of that target is not passed on to higher-level visual processing systems. Object substitution masking, therefore, has provided a 
method to examine reentrant visual processing (Woodman and Luck, 2003).

Four-dot masking is a paradigm that is thought to act through object substitution (Enns and Di Lollo, 1997; Di Lollo et al., 2000; Enns, 2004). In four-dot masking, performance is greatest when the target and the mask have the same onset and offset (effectively cuing the observer to the target). Four-dot masking can occur when both target and mask have the same onset (termed "common-onset masking") and the mask remains visible longer than the target and can also occur when the onset of the mask appears at varying stimulus onset asynchronies (SOAs) relative to the target (Enns and Di Lollo, 2000). Using this second type of four-dot masking in a behavioral study we found impairments in schizophrenia patients (Green et al., 2011). Moreover, it did not appear that the patients' deficit was due to issues in initial processing of the target because when using the same SOAs and using a single dot to indicate which target to identify, we found: (1) that there was no group difference in behavior; and (2) iconic decay of the target was not different between groups. Therefore, the increased masking effects in schizophrenia patients appeared to be due to the presence of the mask and not other factors. However, we could not determine with a behavioral task if deficits in schizophrenia patients in the object formation stage of visual processing contributed to the deficits assessed with four-dot masking. Indeed, the version of four-dot masking that we used (with delayed-onset mask) could have involved some early masking of object formation, in addition to its characteristic object substitution masking. With the excellent temporal resolution of electroencephalography (EEG) and event-related potentials (ERPs) it is possible to evaluate perceptual and post-perceptual stages separately.

Specific ERP waveform components track with stages of visual information processing, including the visual P100 and N100 and the visual awareness negativity (VAN). The $\mathrm{P} 100$ is a positive wave peaking approximately $100 \mathrm{~ms}$ post stimulus onset. Several neural generators of the P100 have been identified, with activity largest over dorsal stream sites, with extrastriate and striate contributions (Maier et al., 1987; Aine et al., 1995; Di Russo et al., 2001; Vanni et al., 2004). The N100 is a negative wave peaking approximately $150 \mathrm{~ms}$ post stimulus onset. Multiple generators have also been identified for the N100, with the largest activity seen in ventral stream structures such as the object-sensitive lateral occipital complex (LOC; e.g., Bentin et al., 1999; Doniger et al., 2000, 2001, 2002). The VAN has been reported to appear as a relative increase in negativity to conscious vs. unconscious (e.g., correct vs. incorrect) stimuli appearing approximately $200-300 \mathrm{~ms}$ in occipito-temporal sites, though there is considerable variability as to when this component peaks (for a review, see (Railo et al., 2011). Moreover, this ERP component has been proposed as a measure of reentrant cortical activity (Koivisto et al., 2006; Wilenius and Revonsuo, 2007) because of its regional distribution, it is associated with awareness of a visual target and its latency falls within the time frame of when reentrant cortical activity is thought to occur (Lamme and Roelfsema, 2000; Di Russo et al., 2001).

Several ERP studies have examined backward masking in healthy participants. Fahrenfort et al. (2007) found that both masked and unmasked targets produced a strong bilateral anterior occipito-temporal activation that occurred prior to $110 \mathrm{~ms}$ (likely consistent with the visual P100), which the authors attributed to feedforward processing of visual stimuli. However, only unmasked trials resulted in activity between $110-140 \mathrm{~ms}$ at posterior occipital sites, with no activity for masked trials seen at this time frame. The authors interpreted these findings as reflecting reentrant processing being interrupted by the presence of the mask. With a backward masking procedure similar to Fahrenfort et al; Van Loon et al, (2012) found that masking had no effect on the earliest ERP components (i.e., $<120 \mathrm{~ms}$, corresponding to the P100) but effectively decreased later ERP components (i.e., $>150 \mathrm{~ms}$, corresponding to the N100). Moreover, only the later, N100-like response correlated with behavior, such that greater accuracy correlated with a larger N100.

To date, only a handful of ERP studies of four-dot masking have been published (Woodman and Luck, 2003; Reiss and Hoffman, 2006; Kotsoni et al., 2007; Prime et al., 2011) and these studies evaluated whether four-dot masking is consistent with object substitution. In summary, these studies found that feedforward processing of an object is left intact, but later reentrant processing of the target is interrupted due to the mask substituting the target during this stage.

In the current study, we tracked the time course of visual information processing during object substitution masking in a large sample of schizophrenia patients and healthy controls. We manipulated visibility of the targets using various SOAs, such that performance is best at an SOA of $0 \mathrm{~ms}$, with performance decreasing at higher SOAs. We chose this method of delayed-onset masking, rather than common-onset masking, to more directly compare the results of the current study to other masking paradigms commonly used in schizophrenia research that have delayed-onset masks (e.g., see Green et al., 2011). We used ERPs to assess early and later perceptual stages (P100 and N100) and the VAN to examine activity thought to be directly related to reentrant processing. We used the exact same paradigm as in our behavioral study that showed patient-control differences, though sampled a fewer number of SOAs. Based on prior EEG studies of backward masking, we hypothesized that the VAN would be the first component sensitive to object substitution masking. In particular, we expected that in healthy controls a larger VAN to correct vs. incorrect responses would be seen, whereas patients would not exhibit this effect.

\section{MATERIALS AND METHODS PARTICIPANTS}

Most participants also participated in a behavioral study of object substitution in a separate session and using a somewhat different procedure from the one presented in the current paper (Green et al., 2011). Seventy-seven stabilized outpatients with schizophrenia and 66 healthy control subjects participated in the study. One patient was excluded from analysis due to having an insufficient amount of usable EEG data (see below). Thus, the final patient sample size was $n=76$. Patients were recruited from outpatient treatment clinics at the Veterans Affairs Greater Los Angeles Healthcare System (VAGLAHS) and through presentations at community residences. Patients met criteria based on the Structured Clinical Interview for DSM-IV Axis I Disorders (First et al., 1997). Sixty-two patients were receiving atypical antipsychotic medications, seven were receiving typical antipsychotic 
medications, three were receiving both types of medication, and four were not taking an antipsychotic medication at time of assessment.

Healthy control participants were recruited through internet and newspaper advertisements. Control participants were screened with the SCID and SCID-II (First et al., 1996) and were excluded if they met criteria for any lifetime psychotic disorder; bipolar mood disorder; recurrent depression; substance dependence; paranoid, schizotypal, or schizoid personality disorder; or any evidence (according to participant report) of a history of psychotic disorder among their first-degree relatives.

Additional exclusion criteria for both groups included being younger than 18 or older than 60 years, diagnosed with an active substance use disorder in the past 6 months, any identifiable neurological disorder, mental retardation, history of loss of consciousness for more than $1 \mathrm{~h}$, or insufficient fluency in English. All participants had the capacity to give informed consent and provided written informed consent after all procedures were explained in accordance with procedures approved by the Institutional Review Boards at UCLA and VAGLAHS.

\section{CLINICAL RATINGS}

Psychiatric symptoms during the previous month were rated using the 24-item UCLA version of the Brief Psychiatric Rating Scale (BPRS; Overall and Gorham, 1962; Lukoff et al., 1986) and the Scale for the Assessment of Negative Symptoms (SANS; Andreasen, 1984) by a trained rater. For the BPRS we report the "positive symptom" and "depression/anxiety" factors (Kopelowicz et al., 2008); for the SANS we report the global scores for affective flattening, alogia, anhedonia, and avolition (Table 1). All clinical assessments were conducted by interviewers trained to reliability through the Treatment Unit of the Department of Veterans Affairs VISN 22 Mental Illness Research, Education, and Clinical Center (MIRECC) based on previously reported procedures (Ventura et al., 1993, 1998).

\section{BACKWARD MASKING TASK}

All stimuli were presented using E-Prime 1.1 (Psychological Software Tools, Pittsburgh, PA) on a $17^{\prime \prime}$ cathode ray tube monitor running at a $160 \mathrm{~Hz}$ refresh rate. Participants sat $1 \mathrm{~m}$ away from the monitor.

The four-dot masking procedure was modified from similar tasks described elsewhere (Enns and Di Lollo, 2000; Enns, 2004) and was based on a larger behavioral study of four-dot masking from our laboratory (Green et al., 2011). In the task, four potential targets, consisting of four squares with a notch missing from the top, bottom, or side, appeared in a notional square on the monitor. Following the target stimuli, a mask was presented which comprised four-dots arranged in a square that surrounded, but did not touch, one of the potential targets (see Figure 1). The mask cued the location of the target. Each potential target measured $1.55^{\circ} \times 1.55^{\circ}$ of visual angle and was arranged in a square of $4.58^{\circ} \times 4.58^{\circ}$ of visual angle. The four-dot mask measured $2.23^{\circ} \times 2.23^{\circ}$ of visual angle and each dot in the mask subtended $0.23^{\circ} \times 0.23^{\circ}$ of visual angle. The target array was presented for $25 \mathrm{~ms}$ and the mask was presented for $37.5 \mathrm{~ms}$. All stimuli were suprathreshold, with stimuli presented in black
Table 1 | Demographic information and symptom ratings.

\begin{tabular}{|c|c|c|c|c|}
\hline & \multicolumn{2}{|c|}{$\begin{array}{l}\text { Patients } \\
(n=76)\end{array}$} & \multicolumn{2}{|c|}{$\begin{array}{l}\text { Normal controls } \\
(n=66)\end{array}$} \\
\hline & Mean & SD & Mean & SD \\
\hline Age $^{*}$ & 46.3 & 10.1 & 37.7 & 9.8 \\
\hline Parental education & 12.8 & 2.9 & 13.5 & 2.5 \\
\hline Percent female & $20 \%$ & & $27 \%$ & \\
\hline \multicolumn{5}{|l|}{ BPRS } \\
\hline Total score & 43.6 & 9.9 & & \\
\hline \multicolumn{5}{|c|}{ Factors (mean score per item) } \\
\hline Depression/anxiety & 1.9 & 0.7 & & \\
\hline Positive symptoms & 2.2 & 0.9 & & \\
\hline \multicolumn{5}{|l|}{ SANS global scores } \\
\hline Affective flattening & 1.6 & 1.3 & & \\
\hline Alogia & 0.7 & 1.0 & & \\
\hline Avolition & 2.2 & 1.4 & & \\
\hline Anhedonia & 2.7 & 1.8 & & \\
\hline
\end{tabular}

${ }^{*} p<0.01$, for difference between controls and patients.

presented on a white background. The luminance of the background was $260 \mathrm{~lx}$ while that of the stimuli was $9 \mathrm{~lx}$, resulting in a contrast of $\sim 93 \%$, defined by Michelson's contrast: contrast $=\left(L_{\max }-L_{\min }\right) /\left(L_{\max }+L_{\min }\right)$. We collected data on four target-mask stimulus onset asynchronies (SOAs: 0, 50, 100, and $150 \mathrm{~ms}$ ). However, we only analyzed data from the first three SOAs, as the mask terminated on or before the ERP components of interest for these SOAs, and there was little difference in performance between SOAs of 100 and 150. Fifty-four trials per SOA were presented in quasi-randomized fashion (18 trials for each of the three potential sides where the notch appears).

Each trial started with a fixation cross presented for $450 \mathrm{~ms}$ followed by a blank screen for $500 \mathrm{~ms}$. Target and mask stimuli, separated by the SOAs mentioned above, were then presented. A $1 \mathrm{~s}$ blank screen was then presented, followed by a prompt for the participant to make a response. Participants verbally reported the direction of the notch in the target and the experimenter entered the response and initiated the next trial. The total number of correct responses (out of 54) at each SOA was analyzed.

\section{EEG RECORDING}

Electroencephalography was continuously recorded using a 64channel Neuroscan SynAmps2 amplifier and a Neuroscan 64channel QuickCap (Compumedics USA, Charlotte, NC). Data were sampled at $500 \mathrm{~Hz}$ with a bandpass of $1-100 \mathrm{~Hz}$. Horizontal electrooculogram (EOG; placed on the outer canthus of the left and right eye) and vertical EOG (placed above and below the left eye) was also recorded. The reference during recording was a point halfway between electrodes $\mathrm{Cz}$ and $\mathrm{CPz}$, and all sites were re-referenced offline to the average of the left and right mastoids. An electrode affixed to the forehead served to ground the array.

\section{ERP DATA ANALYSIS}

Data were processed offline using Neuroscan Scan 4.3 and BrainVision Analyzer 2 software (Brain Products, Gilching, Germany). 


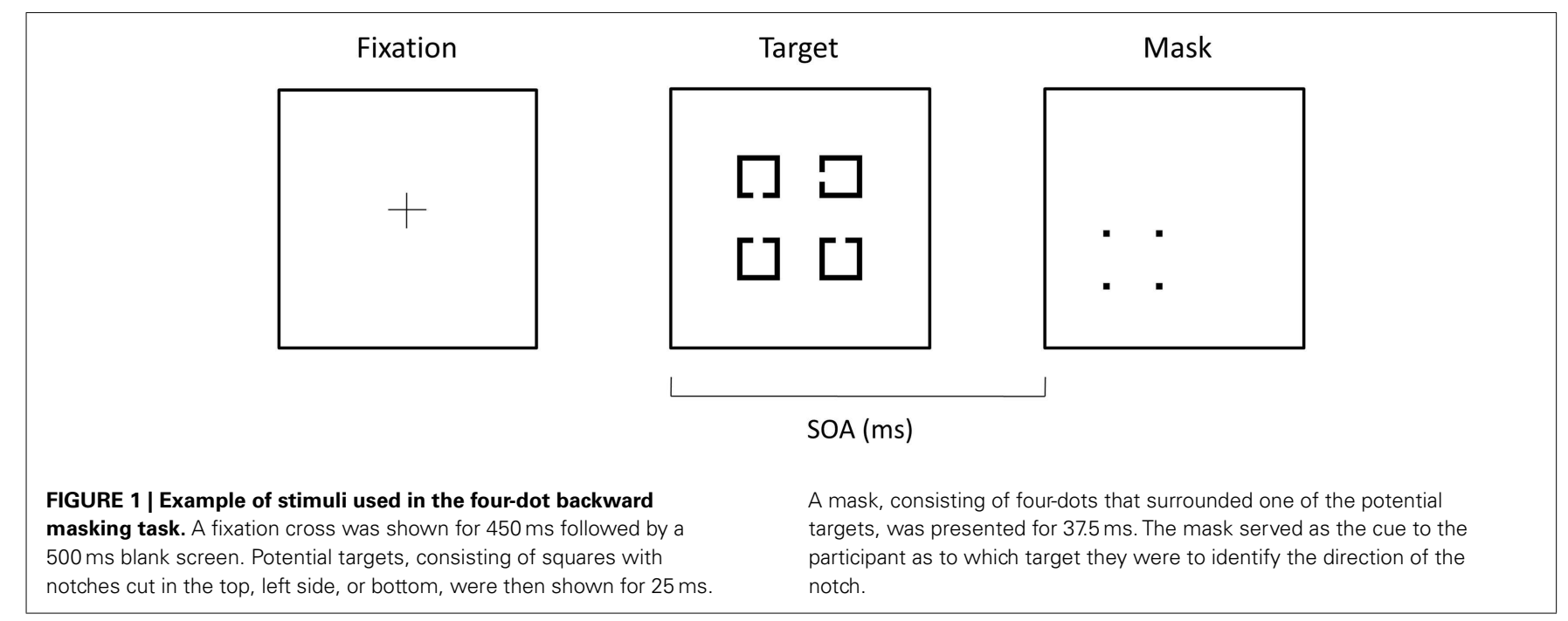

Vertical eye blinks were removed using a regression-based algorithm (Semlitsch et al., 1986). Data were low-pass filtered at $30 \mathrm{~Hz}$, with a $24 \mathrm{~dB}$ roll off zero phase shift filter. Data were epoched to $100 \mathrm{~ms}$ pre- and $700 \mathrm{~ms}$ post-target onset. Baseline correction from the $100 \mathrm{~ms}$ prior to stimulus presentation was applied. Epochs containing activity that exceeded $\pm 100 \mu \mathrm{V}$ were rejected at the sites that were used for data analysis (P7, P5, P6, P8, PO7, PO8, O1, $\mathrm{O} 2$ ). Visual inspection of trials was then performed to eliminate any remaining abnormal EEG responses. The mean number of valid trials per SOA (out of a total of 54) included in subsequent statistical analyses was 51.7 (range for individual participants, 2954 ) and 52.4 (range for individual participants, 44-54) for patients and controls, respectively.

Event-related potentials were created by averaging together all accepted trials (regardless of accuracy), separately for each SOA. A time window was defined for each ERP component based on the peak activity observed by inspection of the mean global field power averaged across all subjects and SOAs. The width of the time window was selected to ensure coverage of each component and the mean activity within each window was the main dependent measure. The time windows were 68-108 and 134-174 ms for the P100 and N100, respectively. The P100 and N100 were examined in six parieto-occipital electrode sites where visual ERPs were largest based on visual inspection of the waveforms (P7, PO7, P5, P8, PO8, and P6). These sites were also chosen as they overlap with sites used in previous examinations of the N100 and P100 response in visual processing studies in schizophrenia (e.g., Butler et al., 2007). Activity was examined separately for each hemisphere by averaging the three left and the three right electrodes.

The VAN was analyzed in 50 patients and 43 healthy controls that had at least 15 artifact-free epochs for both correct and incorrect responses at each SOA of 50 and $100 \mathrm{~ms}$. For patients, there were a mean (SD) 24.1 (5.6) and 28.0 (5.5) trials for correct and incorrect, respectively, averaged over the two SOAs; for controls there were 28.6 (6.2) and 24.2 (6.1) trials for correct and incorrect, respectively. Activity at electrodes $\mathrm{PO} 7, \mathrm{PO} 8, \mathrm{O} 1$, and $\mathrm{O} 2$ were examined for the VAN. The VAN was measured as the mean activity within the time window of 250-310 ms. Additionally, the P100 and N100 (using the same time windows and electrodes described above) to correct and incorrect responses were examined.

\section{DATA ANALYSIS}

For demographic data, group differences were evaluated with $t$ tests and chi-square tests. 2 (group) $\times 2$ (hemisphere) $\times 3$ (SOA) repeated measures ANOVAs were run separately for P100 and N100 for the entire sample, disregarding accuracy. To examine the VAN and accuracy effects on the P100 and N100 in the subsample, separate 2 (group) $\times 2$ (accuracy) $\times 2$ (SOA) repeated measures ANOVAs were run. In cases of repeated measures with more than one degree of freedom, we used Greenhouse-Geisser correction factors $(\varepsilon)$. We report the uncorrected degrees of freedom, the corrected $p$-value, partial eta-square effect sizes $\left(\eta_{p}^{2}\right)$, and $\varepsilon$. All statistical analyses used a two-tailed significance level of 0.05 . Bonferroni-corrected multiple comparisons were used to ensure a family wise significance level of $p<0.05$.

\section{RESULTS \\ DEMOGRAPHICS}

Demographic information is presented in Table 1. Patients were clinically stable with relatively low levels of symptoms. They were significantly older than controls but did not differ in terms of gender or parental education. As we have shown in our previous studies, age is significantly correlated with backward masking performance (Green et al., 2011). Age is also largely correlated with performance in the current study for both groups, with $r$ 's between -0.30 and -0.38 for each SOA. Therefore, we included age as a covariate in our analyses. We report age-corrected means (standard deviations) where appropriate.

\section{BEHAVIOR}

Results of the analysis revealed a significant main effect of group $\left(F_{1,139}=19.76, p<0.001, \eta_{p}^{2}=0.12\right)$ and a significant main effect of SOA $\left(F_{2,278}=8.48, p<0.001, \varepsilon=0.92, \eta_{p}^{2}=0.06\right)$. The group by SOA interaction was not significant $(p>0.17)$. Overall, patients showed poorer performance compared to controls and both groups showed the expected decline in accuracy as SOA increased (see Figure 2). 


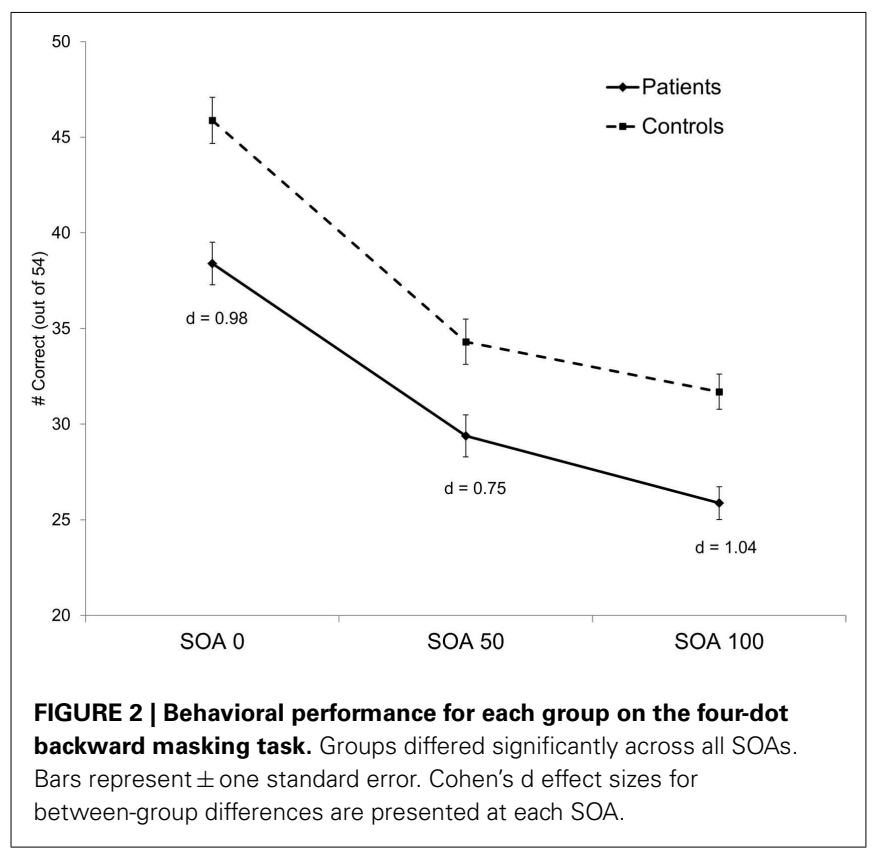

EEG

Means and SD for the two ERP components can be seen in Table 2. Figure 3 shows the ERP waveforms (averaged over the six parietooccipital electrodes mentioned above) at each SOA for each group. Figure 4 shows the VAN waveforms (averaged over the two SOAs and the four parieto-occipital electrodes mentioned above) for each group.

\section{P100}

There was only a significant effect for group $\left(F_{1,139}=3.94\right.$, $\left.p<0.05, \eta_{p}^{2}=0.03\right)$. There were no other significant main effects or interactions. Controls had a larger P100 compared to patients [1.92 (1.26) versus $1.41(1.25) \mu \mathrm{V}$, respectively].

\section{N100}

Results of the analysis revealed a significant main effect of group $\left(\mathrm{F}_{1,139}=5.42, p<0.03, \eta_{p}^{2}=0.04\right)$ and a significant group $\times$ SOA interaction $\left(\mathrm{F}_{2,278}=7.81, p<0.001, \varepsilon=0.98, \eta_{p}^{2}=\right.$ $0.05)$. Controls had a significantly larger N100 compared to patients $[-2.86(2.13)$ versus $-1.77(2.01) \mu \mathrm{V}]$. Controls showed significantly larger N100 amplitudes at all SOAs, except at SOA 50 , compared to the patients. The group $\times$ SOA interaction was due to patients showing an increase in amplitude at SOA 50 whereas controls show a decrease, a pattern difference that was not predicted.

\section{EFFECTS OF ACCURACY ON ERPs AT SOAs 50 AND $100 \mathrm{~ms}$ $P 100$}

The main effects of group, accuracy, and SOA were not significant; there were also no significant interactions, all $F$ 's $<1.40$, $p$ 's $>0.24$.

\section{N100}

The main effects of group, accuracy, and SOA were not significant. There was only a significant group $\times$ SOA interaction
Table 2 | Mean (SD) amplitudes for each group and each ERP component.

\begin{tabular}{lllll}
\hline & \multicolumn{2}{c}{ SOA 0 } & SOA 50 & SOA 100 \\
\hline P100 & Patients & & & \\
& $\mathrm{L}$ & $1.37(1.56)$ & $1.24(1.57)$ & $1.38(1.52)$ \\
& $\mathrm{R}$ & $1.68(1.68)$ & $1.66(1.74)$ & $1.75(1.79)$ \\
& Controls & & & \\
& $\mathrm{L}$ & $1.89(1.57)$ & $1.79(1.59)$ & $1.70(1.54)$ \\
$\mathrm{N} 100$ & $\mathrm{R}$ & $2.03(1.69)$ & $1.98(1.76)$ & $1.89(1.81)$ \\
& Patients & & & \\
& $\mathrm{L}$ & $-1.76(2.43)$ & $-1.93(2.33)$ & $-1.60(2.23)$ \\
& $\mathrm{R}$ & $-2.19(2.54)$ & $-2.09(2.53)$ & $-1.85(2.40)$ \\
& Controls & & & \\
& $\mathrm{L}$ & $-2.55(2.46)$ & $-2.38(2.36)$ & $-2.67(2.25)$ \\
& $\mathrm{R}$ & $-3.09(2.57)$ & $-2.74(2.56)$ & $-3.08(2.43)$ \\
\end{tabular}

$L$, left; $R$, right.

$\left(\mathrm{F}_{1,90}=11.25, p<0.001, \eta_{p}^{2}=0.11\right)$. The group $\times$ SOA interaction was due to patients and controls having similar amplitudes at an SOA of 50, whereas controls had a larger amplitude than patients at an SOA of $100 \mathrm{~ms}$.

\section{VAN}

As the VAN appears as a greater difference between correct vs. incorrect responses, we expected to see a significant difference in the healthy controls, and we did. For controls, the difference between correct and incorrect reached significance $\left(\mathrm{t}_{42}=2.47\right.$, $p<0.02), 0.27(1.25)$ vs. $0.59(1.15) \mu \mathrm{V}$, correct vs. incorrect respectively (effect size $=0.27$ ). In contrast, for the patients the $t$-tests showed no significant difference for correct vs. incorrect responses in patients $\left(\mathrm{t}_{49}=0.55, p<0.60\right),-0.15(0.94)$ vs. -0.08 (1.03) $\mu \mathrm{V}$, correct vs. incorrect respectively (effect size $=0.07$ ). The group $\times$ accuracy interaction failed to reach significance $\left(\mathrm{F}_{1,90}=2.18, p<0.15, \eta_{p}^{2}=0.02\right)$. Hence, although group differences in VAN were predicted, they need to be interpreted with caution given the non-significant group by accuracy interaction.

\section{RELATIONSHIP BETWEEN ERPS AND BEHAVIOR}

Correlations were examined between the P100 and N100 and behavioral performance at each SOA for each group, controlling for age. P100 and N100 activity was averaged across hemisphere for each SOA. As can be seen in Table 3, P100 amplitude was largely uncorrelated with performance in both groups (only one of the eight correlations reached significance). N100 amplitude correlated significantly with performance at the two later SOAs (where masking effects occurred), but only in the healthy controls. P100 was significantly correlated with $\mathrm{N} 100$ at each respective SOA in both groups, $r$ 's ranging from -0.50 to -0.33 .

\section{AGE EFFECTS}

Because analyses of covariance can sometimes misrepresent relationships among variables, we considered the potential effect of age on the behavioral and ERP data, without including a covariance analysis. We performed a median split, creating a "young" patient group $(n=39$, mean age $=38.6)$ and an "old" patient 
Schizophrenia Patients

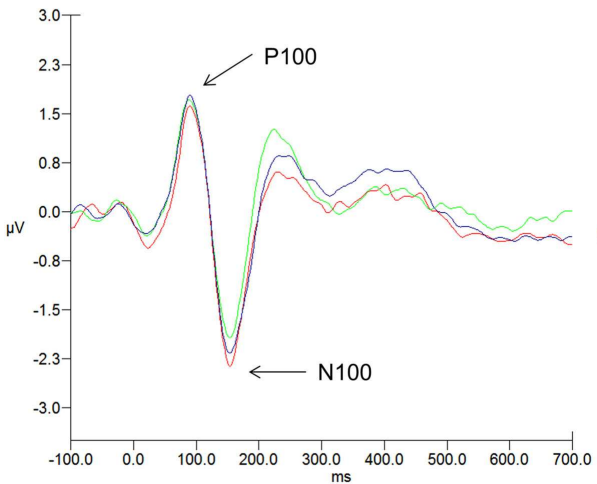

Healthy Controls

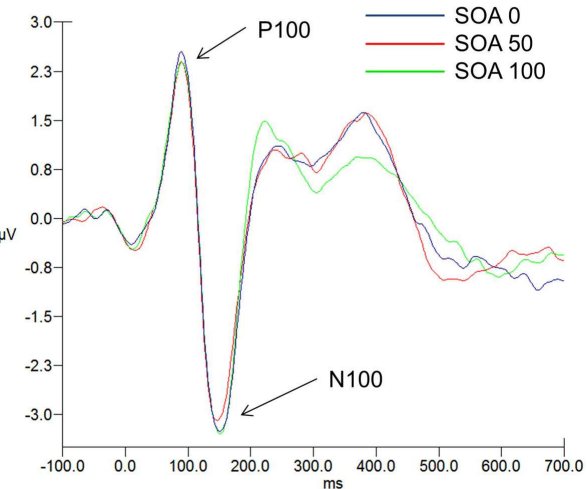

FIGURE 3 | ERPs time-locked to target onset (0 ms) for both groups at each SOA. The P100 and N100 (averaged over electrodes P7, PO7, P5, P6, PO8, P8) are noted by arrows. SOA $0 \mathrm{~ms}$, dark blue; SOA $50 \mathrm{~ms}$, red; SOA $100 \mathrm{~ms}$, green.

Schizophrenia Patients

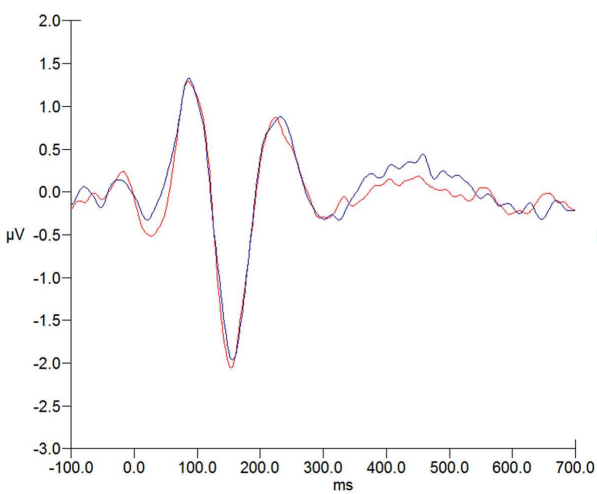

Healthy Controls

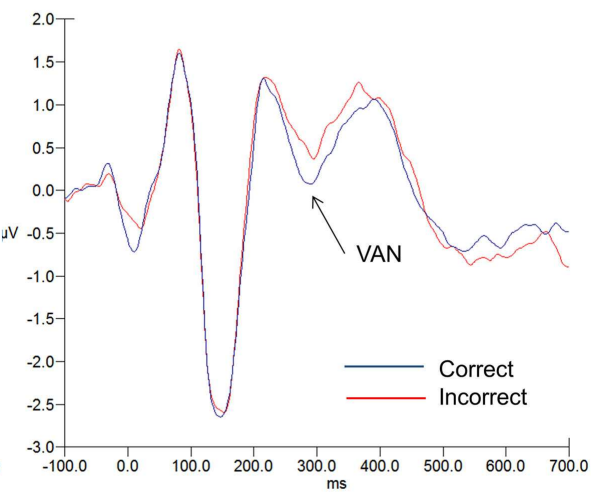

FIGURE 4 |VAN waveforms for correct (blue) and incorrect (red) responses for schizophrenia patients (on the left) and healthy controls (on the right). The VAN was averaged over SOAs 50 and 100 (averaged over electrodes PO7, PO8, 01, O2).

Table 3 | Correlations between backward masking performance (\# targets correctly identified) and ERP components.

\begin{tabular}{lrrr}
\hline & SOAO & SOA50 & SOA100 \\
\hline \multicolumn{2}{l}{ Schizophrenia patients } & & \\
P100 & 0.10 & 0.10 & 0.13 \\
N100 & -0.19 & -0.13 & -0.03 \\
Healthy controls & & & \\
P100 & 0.09 & 0.09 & $0.29^{*}$ \\
N100 & -0.14 & $-0.38^{*}$ & $-0.31^{*}$
\end{tabular}

${ }^{*} p<0.05$

group $(n=37$, mean age $=54.4)$. The "young" patient group more closely matched the age of the healthy control group, with no statistical difference in age between these two groups. Two analyses were conducted. First, differences in ERPs and behavior between the "young" and "old" schizophrenia patient groups were examined; no significant group effects or interactions with group were seen (all F's $<1.0$, p's $>0.4$ ). Second, differences in ERPs and behavior between only the "young" patients and the healthy controls were examined. Similarly, we performed this same split for the VAN analyses, with a "young" patient group $(n=25$, mean age $=40.0)$ and an "old" patient group $(n=25$, mean age $=50.5)$ being created. Again, there were no difference between the "young" and "old" patient groups and the pattern of results remained the same comparing the "young" patients to the healthy control sample. These analyses revealed the exact same pattern of results as when using the entire patient sample, indicating the presence of behavioral and ERP differences between schizophrenia patients and healthy controls regardless of age.

\section{DISCUSSION}

Using ERPs, we were able to clarify the nature of the patients' behavioral deficit in four-dot object substitution masking. Several key findings emerged from this study. First, patients exhibited 
significantly reduced P100 and N100 amplitudes compared to controls across all SOAs, indicating impairment in perceptual processes across levels of visibility. Second, N100 correlated with accuracy in controls, but not patients, at SOAs where masking occurs. Third, controls, but not patients, exhibited a VAN response, though the group by accuracy interaction did not reach significance. These results provide further evidence that schizophrenia patients likely have a dysfunction in reentrant visual processing.

The reduced amplitude of P100 and N100 is consistent with previous reports of EEG abnormalities during early visual perception in schizophrenia (Foxe et al., 2001; Doniger et al., 2002; Schechter et al., 2005), suggesting dysfunction at the earliest stages of object formation. However, it seems unlikely that the P100 component directly affected object substitution for either group (i.e., amplitudes did not change with changing visibility). As mentioned in the introduction, ERP studies of backward masking show no effect on the P100, indicating that feedforward processing is left relatively intact. Furthermore, in the accuracy analysis of the ERP data, there was no effect of accuracy on the P100 and the subgroup of patients and controls included in the accuracy analyses did not differ in P100 amplitude. We found a somewhat mixed pattern for the effects of object substitution masking on the N100, in that there were no effects of SOA (i.e., visibility) and no effect of accuracy on N100 amplitude. On the other hand, there was a correlation between behavior and amplitude in the controls that may simply reflect greater attentional effort devoted to processing stimuli that were harder to accurately detect.

The finding of the lack of a VAN to correct vs. incorrect trials in schizophrenia patients points to dysfunctional reentrant processing. As mentioned in the introduction, the VAN appears to be the first ERP correlate of visual consciousness and likely is a direct measure of reentrant processing. Moreover, there was no effect of accuracy on either the P100 or N100, reflecting that these components are unlikely related to reentrant processing. The healthy controls exhibited a VAN, indicating that effective four-dot masking in this study interrupted reentrant visual processing. Given the lack of this finding in the schizophrenia patients, these results imply that schizophrenia patients' deficit during object substitution may be due to a type of dysfunction (e.g., lack of neural synchronization, coherence, etc.) in reentrant processing along this pathway. Regardless of how patients correctly identified stimuli in the current study, the VAN results suggest a dysfunction in reentrant processing. However, the lack of a significant group $\times$ accuracy interaction in the VAN data means that we interpret these findings with caution.

There also remains the possibility that other processes, such as consciousness of stimuli and attentional effort, may be accounting for our behavioral and EEG findings. For example, Del Cul et al. (2006) found that schizophrenia patients' ability to consciously process targets in the presence of a mask was diminished in comparison to healthy controls; however, their ability to subliminally process the targets was intact. The authors interpreted their findings as showing that patients' feedforward processing during masking was intact whereas conscious processing of those stimuli are dysfunctional. Also, Lalanne et al. (2012) suggested that backward masking impairments in schizophrenia patients may be due to a lack of focused attention on the target. Finally, using pupillometry during a backward masking task, Granholm et al. (2009) found that impaired performance in patients was attributable to abnormalities in attentional resource availability. These other factors may be alternative possibilities for the findings, or constitute explanations at other levels of processing.

As with our behavioral study (Green et al., 2011), patients' behavioral performance was worse than controls across all SOAs, including at the earliest SOA that has minimal masking. This finding limits interpretation in that it could be that patients are unable to correctly identify the target regardless of the effectiveness of the mask. We were able to address this possibility in our previous behavioral study which included a cuing procedure (see Introduction). For example, four-dot masking tasks depend on iconic decay (the visible persistence of a target) and group differences in this process could account for the masking deficit. However, we examined iconic decay in our behavioral study using a simple cuing task and found no differences between the patients and controls on iconic decay. Thus, differences in decay rates are unlikely to account for the performance differences. The current paradigm had no similar control procedure, and that is one limitation of the study.

The current study has other limitations. First, nearly all patients were medicated at the time of testing, potentially affecting the results. However, there is evidence that antipsychotic medication does not affect masking performance (Cadenhead et al., 1997; Green et al., 1999; Butler et al., 2003) or visual ERPs (Butler et al., 2001; Schechter et al., 2005), making medication effects unlikely. Second, our delayed-onset paradigm might not have completely eliminated the interrupting effect of the onset of the transient channels elicited by the mask (Jannati et al., 2011). While a common-onset masking procedure (Enns and Di Lollo, 2000) might be a more complete way to isolate reentrant processing during masking, there remains considerable evidence that even in metacontrast masking reentrant processing is being interrupted (Di Lollo et al., 2000; Breitmeyer, 2007; Fahrenfort et al., 2007). Therefore it is likely that our behavioral and VAN results reflect in a dysfunction in reentrant processing in patients with schizophrenia.

Another limitation is that our design was not optimized for eliciting P300 or N2pc that have been examined in the four-dot backward masking studies conducted in healthy controls (Woodman and Luck, 2003; Prime et al., 2011). Specifically, we did not have a rare stimulus, we had SOAs that were closely spaced, and we did not have lateralized presentation of the stimuli that would have enabled us to collect these other components. A small P300-like component is visible for the shortest SOA in Figure 3, but due to our parameters we did not consider it to be a valid P300. Finally, we did not equate the groups for their "unmasked" target performance (i.e., performance at an SOA of $0 \mathrm{~ms}$ ), as we have done in our previous backward masking studies (Green et al., 2002, 2003, 2006).

The results from this study help clarify findings from our previous behavioral study on object substitution masking (Green et al., 2011). It was not possible to determine from the behavioral study (particularly given our choice of a delayed-onset mask) 
whether the deficits in the patients' identification of targets were due to impairment at the earliest stage of object formation or to impairment in a later stage where reentrant processes are necessary to refine the visual percept. The current study offers tentative support for the idea that impairment of visual processing was attributable to deficits associated with reentrant processing after the initial feedforward sweep. While there is abundant evidence, from our laboratory and others, that dysfunctional bottom-up processes in schizophrenia affect downstream processing of higher-level tasks (Sergi and Green, 2003; Leitman et al., 2005; Dias et al., 2011; Pinheiro et al., 2012), our findings with this paradigm implicate a slightly later visual processing stage.

\section{REFERENCES}

Aine, C. J., Supek, S., and George, J. S. (1995). Temporal dynamics of visual evoked neuromagnetic sources: effects of stimulus parameters and selective attention. Int. J. Neurosci. 80, 79-104.

Andreasen, N. C. (1984). The scale for the assessment of negative symptoms (SANS). Iowa City, IA: The University of Iowa.

Bentin, S., Mouchetant-Rostaing, Y., Giard, M. H., Echallier, J. F., and Pernier, J. (1999). ERP manifestations of processing printed words at different psycholinquistic levels: time course and scalp distribution. J. Cogn. Neurosci. 11, 235-260.

Breitmeyer, B. G. (2007). Visual masking: past accomplishments, present status, future developments. $A d v$. Cogn. Psychol. 3, 9-20.

Butler, P. D., Desanti, L. A., Maddox, J., Harkavy-Friedman, J. M., Amador, X. F., Goetz, R. R., et al. (2003). Visual backward-masking deficits in schizophrenia: Relationship to visual pathway function and symptomatology. Schizophr. Res. 59, 199-209.

Butler, P. D., and Javitt, D. C. (2005). Early-stage visual processing deficits in schizophrenia. Curr. Opin. Psychiatry 18, 151-157.

Butler, P. D., Martinez, A., Foxe, J. J., Kim, D., Zemon, V., Silipo, G., et al. (2007). Subcortical visual dysfunction in schizohprenia drives secondary cortical impairments. Brain 130, 417-430.

Butler, P. D., Schechter, I., Zemon, V., Schwartz, S. G., Greenstein, V. C., Gordon, J., et al. (2001). Dysfunction of early-stage visual processing in schizophrenia. Am. J. Psychiatry 158, 1126-1133.

Butler, P. D., Zemon, V., Schechter, I., Saperstein, A. M., Hoptman, M. J., Lim, K. O., et al. (2005). Early-stage visual processing and cortical amplification deficits in schizophrenia.
Arch. Gen. Psychiatry 62 495-504.

Cadenhead, K. S., Geyer, M. A., Butler, R. W., Perry, W., Sprock, J., and Braff, D. L. (1997). Information processing deficits of schizophrenia patients: Relationship to clinical ratings, gender and medication status. Schizophr. Res. 28, 51-62.

Chen, Z., and Treisman, A. (2009). Implicit perception and level of processing in object-substitution masking. Psychol. Sci. 20, 560-567.

Del Cul, A., Dehaene, S., and Leboyer, M. (2006). Preserved subliminal processing and impaired conscious access in schizophrenia. Arch. Gen. Psychiatry 63, 1313-1323.

Di Lollo, V., Enns, J. T., and Rensink, R. A. (2000). Competition for consciousness among visual events: The psychophysics of reentrant visual processes. J. Exp. Psychol. Gen. 129, 481-507.

Di Russo, F., Martinez, A., Sereno, M. I., Pitzalis, S., and Hillyard, S. A. (2001). Cortical sources of the early components of the visual evoked potential. Hum. Brain Mapp. 15, 95-111.

Dias, E. C., Butler, P. D., Hoptman, M. J., and Javitt, D. C. (2011). Early sensory contributions to contextual encoding deficits in schizophrenia. Arch. Gen. Psychiatry 68, 654-664.

Doniger, G. M., Foxe, J. J., Murray, M. M., Higgins, B. A., and Javitt, D. C. (2002). Impaired visual object recognition and dor$\mathrm{sal} /$ ventral stream interaction in schizophrenia. Arch. Gen. Psychiatry 59, 1011-1020.

Doniger, G. M., Foxe, J. J., Murray, G. M., Higgins, B. A., Snodgrass, J. G., Schroeder, C. E., et al. (2000). Activation timecourse of ventral visual stream object-recognition areas: High-density electrical mapping of perceptual closure processes. J. Cogn. Neurosci. 12, 615-621.

Doniger, G. M., Foxe, J. J., Schroeder, C. E., Murray, G. M., Higgins,

\section{ACKNOWLEDGMENTS}

Support for this study came from National Institute of Mental Health grants MH43292 and MH065707 (principal investigator, Dr. Green). Jonathan Wynn was supported by a Veterans Affairs Career Development Award. The funding agencies had no other role in the study. None of the authors have any financial disclosures or conflicts of interest to report. Dr. Wynn takes responsibility for the integrity of the data and the accuracy of the data analyses. All authors had full access to all of the data in the study. The authors thank Daniel Mathalon, M.D., Ph.D. and Brian Roach for helpful comments on previous drafts of the manuscript. The authors thank Cory Tripp, Poorang Nori, and Mark McGee for help in data collection.

B. A., and Javitt, D. C. (2001). Visual perceptual learning in human object recognition areas: a repetition priming study using high-density electrical mapping. Neuroimage 13, 305-313.

Dux, P. E., Visser, T. A., Goodhew, S. C., and Lipp, O. V. (2010). Delayed reentrant processing impairs visual awareness: An object-substitution masking study. Psychol. Sci. 21, 1242-1247.

Enns, J. T. (2004). Object substitution and its relation to other forms of visual masking. Vision Res. 44, 1321-1331.

Enns, J. T., and Di Lollo, V. (1997). Object substitution: A new form of masking in unattended visual locations. Psychol. Sci. 8, 135-139.

Enns, J. T., and Di Lollo, V. (2000). What's new in visual masking? Trends Cogn. Sci. 4, 345-352.

Fahrenfort, J. J., Scholte, H. S., and Lamme, V. A. (2007). Masking disrupts reentrant processing in human visual cortex. J. Cogn. Neurosci. 19, 1488-1497.

First, M. B., Gibbon, M., Spitzer, R. L., Williams, J. B. W., and Benjamin, L. (1996). Structured Clinical Interview for DSM-IV Avis II Personality Disorders. New York: New York State Psychiatric Institute.

First, M. B., Spitzer, R. L., Gibbon, M. and Williams, J. B. W. (1997). Structured Clinical Interview for DSM-IV Axis I Disorders - Patient Edition. New York: New York State Psychiatric Institute.

Foxe, J. J., Doniger, G. M., and Javitt, D. C. (2001). Early visual processing deficits in schizophrenia: impaired P1 generation revealed by highdensity electrical mapping. Neuroreport 12, 3815-3820.

Granholm, E., Fish, S. C., and Verney, S. P. (2009). Pupillometric measures of attentional allocation to target and mask processing on the backward masking task in schizophrenia. Psychophysiology 46, 510-520.

Green, M. F., Lee, J., Cohen, M. S., Engel, S. A., Korb, A. S., Nuechterlein, K H., et al. (2009). Functional neuroanatomy of visual masking deficits in schizophrenia. Arch. Gen. Psychiatry 66, 1295-1303.

Green, M. F., Nuechterlein, K. H., Breitmeyer, B., and Mintz, J. (2006). Forward and backward visual masking in unaffected siblings of schizophrenic patients. Biol. Psychiatry 59, 446-451.

Green, M. F., Nuechterlein, K. H., and Breitmeyer, B. G. (2002). Development of a computerized assessment for visual masking. Int. J. Methods Psychiatr. Res. 11, 83-89.

Green, M. F., Nuechterlein, K. H., Breitmeyer, B. G., and Mintz, J. (1999). Backward masking in unmedicated schizophrenic patients in psychotic remission: possible reflection of aberrant cortical oscillation. Am. J. Psychiatry 156, 1367-1373.

Green, M. F., Nuechterlein, K. H., Breitmeyer, B. G., Tsuang, J., and Mintz, J. (2003). Forward and backward visual masking in schizophrenia: influence of age. Psychol. Med. 33, 887-895.

Green, M. F., Nuechterlein, K. H., and Mintz, J. (1994). Backward masking in schizophrenia and mania: II. Specifying the visual channels. Arch. Gen. Psychiatry 51, 939-944.

Green, M. F., Wynn, J. K., Breitmeyer, B., Mathis, K. I., and Nuechterlein, K. H. (2011). Visual masking by object substitution in schizophrenia. Psychol. Med. 41, 1489-1496.

Jannati, A., Spalek, T. M., and Di Lollo, V. (2011). On the labile memory buffer in the attentional blink: masking the $\mathrm{T} 2$ representation by onset transients mediates the AB. J. Exp. Psychol. Hum. Percept. Perform. 37, 1182-1192. 
Javitt, D. C. (2009). When doors of perception close: bottom-up models of disrupted cognition in schizophrenia. Annu. Rev. Clin. Psychol. 5, 249-275.

Koivisto, M., Revonsuo, A., and Lehtonen, M. (2006). Independence of visual awareness from the scope of attention: an electrophysiological study. Cereb. Cortex 16, 415-424.

Kopelowicz, A., Ventura, J., Liberman, R. L., and Mintz, J. (2008). Consistency of brief psychiatric rating scale factor structure across a broad spectrum of schizophrenia patients. Psychopathology 41, 77-84.

Kotsoni, E., Csibra, G., Mareschal, D., and Johnson, M. H. (2007). Electrophysiological correlates of commononset visual masking. Neuropsychologia 45, 2285-2296.

Lalanne, L., Dufour, A., Despres, O., and Giersch, A. (2012). Attention and masking in schizophrenia. Biol. Psychiatry 71, 162-168.

Lamme, V. A., and Roelfsema, P. R. (2000). The distinct modes of vision offered by feedforward and recurrent processing. Trends Neurosci. 23, 571-579.

Leitman, D. I., Foxe, J. J., Butler, P. D., Saperstein, A., Revheim, N., and Javitt, D. C. (2005). Sensory contributions to impaired prosodic processing in schizophrenia. Biol. Psychiatry 58, 56-61.

Lukoff, D., Nuechterlein, K. H., and Ventura, J. (1986). Manual for the expanded brief psychiatric rating scale. Schizophr. Bull. 12, 578-602.

Maier, J., Dagnelie, G., Spekreijse, H., and van Dijk, B. W. (1987). Principal component analysis for source localization VEPs in man. Vision Res. 27, 165-177.

Overall, J. E., and Gorham, D. R. (1962). The brief psychiatric rating scale. Psychol. Rep. 10, 799-812.
Pinheiro, A. P., Del Re, E., Mezin, J., Nestor, P. G., Rauber, A., McCarley, R. W., et al. (2012). Sensorybased and higher-order operations contribute to abnormal emotional prosody processing in schizophrenia: an electrophysiological investigation. Psychol. Med. 10, 1-16.

Prime, D. J., Pluchino, P., Eimer, M., Dell'acqua, R., and Jolicoeur, P. (2011). Object-substitution masking modulates spatial attention deployment and the encoding of information in visual short-term memory: Insights from occipitoparietal ERP components. Psychophysiology 48, 687-696.

Railo, H., Koivisto, M., and Revonsuo, A. (2011). Tracking the processes behind conscious perception: a review of event-related correlates of visual consciousness. Conscious. Cogn. 20, 972-983.

Rassovsky, Y., Green, M. F., Nuechterlein, K. H., Breitmeyer, B. G., and Mintz, J. (2005). Visual processing in schizophrenia: structural equation modeling of visual masking performance. Schizophr. Res. 78, 251-260.

Rassovsky, Y., Horan, W. P., Lee, J., Sergi, M. J., and Green, M. F. (2011). Pathways between early visual processing and functional outcome in schizophrenia. Psychol. Med. 41, 487-497.

Reiss, J. E., and Hoffman, J. E. (2006). Object substitution masking interferes with semantic processing: evidence from event-related potentials. Psychol. Sci. 17, 1015-1020.

Ro, T., Breitmeyer, B., Burton, P., Singhal, N. S., and Lane, D. (2003). Feedback contributions to visual awareness in human occiptial cortex. Curr. Biol. 13, 1038-1041.

Schechter, I., Butler, P. D., Zemon, V., Revheim, N., Saperstein, A. M., Jalbrzikowski, M., et al. (2005).
Impairments of early-stage transient visual evoked potentials to magnoand parvocellular-selective stimuli in schizophrenia. Clin. Neurophysiol. 116, 2204-2215.

Semlitsch, H. V., Anderer, P., Schuster P., and Presslich, O. (1986). A solution for reliable and valid reduction of ocular artifacts, applied to the P300 ERP. Psychophysiology 23 695-703.

Sergi, M. J., and Green, M. F. (2003). Social perception and early visual processing in schizophrenia. Schizophr. Res. 59, 233-241.

Sillito, A. M., Jones, H. E., Gerstein, G L., and West, D. C. (1994). Featurelinked synchronization of thalamic relay cell firing induced by feedback from the visual cortex. Nature 369, 479-482.

Silverstein, S. M., and Keane, B. P. (2011). Perceptual organization impairment in schizophrenia and associated brain mechanisms: review of research from 2005 to 2010. Schizophr. Bull. 37, 690-699.

Van Loon, A. M., Scholte, H. S., Van Gaal, S., Van Der Hoort, B. J. J., and Lamme, V. a. F. (2012). GABAA agonist reduced visual awareness: a masking-EEG experiment. J. Cogn. Neurosci. 24, 965-974.

Vanni, S., Warnking, J., Dojat, M. Delon-Martin, C., Bullier, J., and Segebarth, C. (2004). Sequence of pattern onset responses in the human visual areas: an fMRI constrained VEP source analysis. Neuroimage 21, 801-817.

Ventura, J., Green, M. F., Shaner, A. and Liberman, R. P. (1993). Training and quality assurance with the brief psychiatric rating scale: 'the drift busters.' Int. J. Methods Psychiatr. Res. 3, 221-224.

Ventura, J., Liberman, R. P., Green, M. F., and Shaner, A. (1998).
Training and quality assurance with the structured clinical interview for DSM-IV. Psychiatry Res 79, 163-173. Wilenius, M. E., and Revonsuo, A. (2007). Timing of the earliest ERP correlate of visual awareness. Psychophysiology 44, 703-710.

Woodman, G. F., and Luck, S. J. (2003). Dissociations among attention, perception, and awareness during object-substitution masking. Psychol. Sci. 14, 605-611.

Wynn, J. K., Light, G. A., Breitmeyer, B. Nuechterlein, K. H., and Green, M. F. (2005). Event-related gamma activity in schizophrenia patients during a visual backward masking task. Am. J. Psychiatry 162, 2330-2336.

Conflict of Interest Statement: The authors declare that the research was conducted in the absence of any commercial or financial relationships that could be construed as a potential conflict of interest.

Received: 30 August 2012; accepted: 14 January 2013; published online: $04 \mathrm{Feb}$ ruary 2013.

Citation: Wynn JK, Mathis KI, Ford J, Breitmeyer BG and Green MF (2013) Object substitution masking in schizophrenia: an event-related potential analysis. Front. Psychology 4:30. doi: 10.3389/fpsyg.2013.00030

This article was submitted to Frontiers in Psychopathology, a specialty of Frontiers in Psychology.

Copyright (C) 2013 Wynn, Mathis, Ford, Breitmeyer and Green. This is an openaccess article distributed under the terms of the Creative Commons Attribution License, which permits use, distribution and reproduction in other forums, provided the original authors and source are credited and subject to any copyright notices concerning any third-party graphics etc. 